\title{
Bernhard Fisseni
}

\section{Grammis' Grammatische Fachbegriffe: Ein Reisebericht}

$\begin{array}{llcl}1 & \text { Aufbruch } & 4.2 & \text { Einschub: Der Dritte W-eg } \\ 2 & \text { Zwei Spaziergänge } & 4.3 & \begin{array}{l}\text { Fernverkehr I: Systematische } \\ \text { Grammatik }\end{array} \\ 2.1 & \text { Erster Weg: Immer der Nase nach } & 4.4 & \begin{array}{l}\text { Fernverkehr II: Grammatik in Fragen } \\ \text { und Antworten }\end{array} \\ & \begin{array}{l}\text { Zweiter Weg: Hypokoristikönchen, } \\ \text { Diatheschen, Sternchen }\end{array} & 5 & \begin{array}{l}\text { Alles eine Frage des Stils, Beispiel: } \\ \text { Beispiele }\end{array} \\ 3 & \begin{array}{l}\text { Vorschläge } \\ \text { Den eigenen Weg finden: O Tempora, } \\ \text { o Modi }\end{array} & 5 & \begin{array}{l}\text { Heimkehr und Schluss } \\ \text { Literatur }\end{array} \\ 4.1 & \begin{array}{l}\text { Ausflüge ins Grüne: Links } \\ \text { Ortsverkehr }\end{array} & 7 & \end{array}$

\begin{abstract}
This article evaluates the terminological component (TC) of the grammis portal on German grammar developed by the Institut fuir Deutsche Sprache. The TC is included into grammis to facilitate nonlinguists ' access to the main components of the portal: Grammar in questions and answers, and the Systematic Grammar. The TC thus has the potential to be an extremely useful and important grammis component. We discuss to what extend the TC achieves its goals, and make some suggestions how it could be improved. The most important aspects considered in the evaluation are: (a) TC completeness and consistency, (b) accessibility and usability of definitions and index, (c) integration of the $\mathrm{TC}$ with the overall system.
\end{abstract}

1 Aufbruch

"grammis®" ${ }^{\circledR}$ ist laut Titelzeile seiner Webs(e)ite „das grammatische informationssystem des ids"; sein Zweck ist laut Leitseite, „die ach so trockene, oft schwer verständliche deutsche Grammatik leichter zugänglich und, soweit möglich, unterhaltsam und interessant zu machen". Das ist ein löbliches Ziel, aber nicht so einfach.

Eine wesentliche Schwierigkeit beim Einstieg in eine Grammatik stellt für LaiInnen natürlich die wuchernde linguistische Terminologie dar. Grammis enthält einen Abschnitt Grammatische Fachbegriffe, der NutzerInnen helfen soll, den Weg durch die beschreibenden Haupt-Module der Systematischen Grammatik und die Datenbank zu Fragen und Antworten zu finden, denn er bietet kurze Definitionen, gelegentlich Beispiele und in Verbindung mit der Grammatischen Ontologie eine Einordnung der Terminologie in den linguistischen Begriffs- und Benennungs-Urwald. Außerdem finden darin sich ein- und aus-

1 Im Rest des Beitrags erscheint Grammis der Lesbarkeit halber mit Majuskel und ohne „®“. 
gehende Verknüpfungen mit der Systematischen Grammatik („Weiterführender Text“) und eingehende aus der Grammatik in Fragen und Antworten.

Der Ansatz ist vielversprechend und weckt Hoffnungen. Wir schildern kurz unseren Eindruck und machen Vorschläge zur Verbesserung.

Hinweis: Den LeserInnen sei versichert, dass die Beispiele nicht mit bösem Willen ausgesucht wurden.

\section{Zwei Spaziergänge}

Wir erkunden die Grammatischen Fachbegriffe anhand zweier kurzer Wege.

\section{$2.1 \quad$ Erster Weg: Immer der Nase nach}

Steigen wir auf der untersten strukturellen sprachlichen Ebene in die Datenbank ein und betrachten den Eintrag für nasal:

(1) Artikulationsart der Konsonanten $/ \mathrm{m} /, \mathrm{n} /$ und $/ \mathrm{n} /$ wie sie z. B. in diesen Wörtern vorkommen:

- Medizin, umgekommen

- Hanna, nichts

- Akzentuierung, Wortstellung

Dem Linguisten erscheint der Eintrag klar und plausibel, aber wie mag es für die Zielgruppe sein? Zunächst wird keine inhaltliche Definition gegeben, etwa: „Bei nasalen Konsonanten entweicht die Luft nicht durch den Mund, sondern durch die Nase." Stattdessen wird von Artikulationsart gesprochen, diese wird in Grammis aber nicht definiert. Überhaupt ist nasal die einzige Artikulationsart, die in der Begriffs-Datenbank erscheint. Auch Konsonant findet sich nicht, dürfte aber wenigstens in Ansätzen bekannt sein (wahrscheinlich etwa: „alle Buchstaben außer $\left.a, e, i, o, u^{\prime \prime}\right)$. Die Phonem-Notation in der Erläuterung dürfte kaum Schaden anrichten, auch wenn man Phonem nicht nachschlagen kann. Das Zeichen,$\eta$ “ überfordert schon manche GermanistInnen und lässt sich höchstens erschlieBen, wenn man den Zusammenhang von Phonemen, Listenpunkten und Fettdruck versteht und - kaum selbstverständlich - von der orthographischen Repräsentation abstrahierend, merkt, dass „ng“ für nur einen Laut steht.

Ein phonologischer oder gar phonetischer Teil ist in der Systematischen Grammatik jedoch (noch?) gar nicht vorgesehen. Wie gerät nasal also unter die Fachbegriffe? Wir spekulieren: Der folgende $\mathrm{Satz}^{2}$ aus der Systematischen Grammatik (Der Formenbestand von

2 Der Satz ist überdies nicht ganz richtig: U.a. wird das Beispiel atm- angeführt - dieser Stamm endet weder auf Dentalplosiv noch auf Doppelnasal, sondern auf „Doppel-(Nasalkonsonant-oderDentalplosiv)“. 
Indikativ und Konjunktiv) ist ohne Kenntnis des Adjektivs nasalisch (verlinkt auf nasal[!]) unverständlich: ${ }^{3}$

(2) Wenn der Stamm auf $<\mathrm{d}>$ oder $<\mathrm{t}>$ oder auf nasalischem[sic!] Doppelkonsonant endet, ist dies [der Einschub von „e“, BF] bei starken und schwachen Verben im Präsens obligatorisch.

Hier wäre u.E. eine Soforthilfe, also die Aufzählung von , $<\mathrm{mm}>$ ", „<nn $>$ “, wie bei „, $<\mathrm{d}>$ “ und , $<\mathrm{t}>$ " hilfreicher gewesen als ein Fachbegriff nasal.

\subsection{Zweiter Weg: Hypokoristikönchen, Diatheschen, Sternchen}

Hypokoristikon* zieht unsere Aufmerksamkeit auf sich: wir kennen es noch nicht. ${ }^{4}$ Dieser Eintrag ist mit einem Sternchen versehen; für solche Einträge gilt:

(3) „Die mit einem Sternchen versehenen Listeneinträge verweisen auf thematisch verwandte Stichwörter (z. B. Ableitung* verweist auf Derivation).“ (Leitseite Grammatische Fachbegriffe)

Im Falle von Hypokoristikon ist das thematisch verwandte Stichwort, zu dem ein erläuternder Eintrag besteht: Diminutivum. Dieser Eintrag erklärt, was ein Diminutiv ist, jedoch nicht die thematische Verwandtschaft zu Hypokoristikon. Folgt man dem Lemma Diathese* zum Eintrag Genus verbi, wird Diathese dort ebenfalls als „thematisch verwandt“ aufgeführt, neben Handlungsform. Es wäre wohl sinnvoll, zwischen semantischer Nähe und (Fast-)Synonymie konsistent zu unterscheiden, denn das Verhältnis von Hypokoristikon zu Diminutiv ist ja ganz anders ${ }^{5}$ als das von Diathese zu Genus Verbi.

Ein anderes Grammis-Modul könnte hier helfen: Die Grammatische Ontologie bietet eine visualisierte Begriffshierarchie und kann so die Verhältnisse zwischen Begriffen einfacher darstellen; auch spielerisches Navigieren in der Ontologie kann Verknüpfungen erschließen, zumal auch die Ontologie meist in die Fachbegriffe verweist. ${ }^{6}$

Die minimale Einheit der Ontologie ist das Termset, das aus mehreren Lemmata bestehen kann. Zum aktuellen Termset werden (Ko-)Hyp- und Hol-/Meronyme sowie „verwandte Termsets" aufgeführt und farblich ${ }^{7}$ codiert dargestellt. Termsets sind offensichtlich keine Synsets im Sinne von WordNet (Fellbaum 1998), sondern nur Mengen irgendwie eng verwandter Begriffe: Sowohl Genus Verbi und Diathese als auch Hypokoristikon und Diminutiv gehören je in ein Termset. Das ist insofern verwirrend, als Hypokoristikon ja ein Hyponym oder verwandter Begriff zu Diminutivum sein müsste. An dieser Stelle kann also leider die Ontologie das Defizit der Definition nicht auffangen.

3 Unterstreichungen stehen für Hyperlinks.

4 Ein Grund für diesen Eintrag ist nicht auszumachen (d.h. weder Fragen und Antworten noch Systematische Grammatik scheinen den Begriff zu enthalten).

5 Wir haben es mittlerweile in einem linguistischen Lexikon nachgeschlagen.

6 Die Möglichkeit, sich in der Ontologie eine Suchanfrage für die anderen Module (ausschließlich der Fachbegriffe) zusammenzustellen, übergehen wir, so schön und anschaulich sie ist.

7 Die benutzten Brauntöne kann der Autor nicht gut identifizieren, falls nur eine Relation angezeigt wird. Sind aber alle Termset-Relationen im Diagramm vorhanden, so sind die Beziehungen leicht auseinanderzuhalten und die Darstellung ist durchaus gefällig. 
Die Begriffsbestände und -hierarchien der beiden Informationsteilsysteme sind offensichtlich auch noch nicht abgeglichen worden: Die Grammatische Ontologie zeigt das Termset nasal nicht als Hyponym zu Artikulationsart, sondern zu distinktives Merkmal. Für letzteres ist ebenfalls kein Eintrag bei den Fachbegriffen zu finden.

\subsection{Vorschläge}

Man sollte hilflose und irreführende Einträge wie Hypokoristikon streichen und die „Termsets“ in der Ontologie auf echte Synonyme beschränken, deren die linguistische Terminologie hinreichend viele bietet - und in Definitionen „verwandte Begriffe“ nach semantischen Relationen trennen.

Es wäre außerdem wünschenswert, dass die begrifflichen Hierarchien der Ontologie und der Fachbegriffe abgeglichen und dass die Einträge so vervollständigt würden, dass das Vokabular zumindest in Richtung der Ontologie-Wurzel abgeschlossen ist und die Definitionen sich (fachlich) auf dieses Vokabular beschränken.

Man kann in den Fachbegriffen suchen; das ist besonders nützlich, wenn eine Datenbank sich im Aufbau befindet und man vielleicht bei einem übergeordneten Eintrag Informationen $\mathrm{zu}$ einem gesuchten Begriff findet. Vorbildlicherweise werden die gefundenen Seiten auch nach Relevanz (,Trefferquote“) sortiert.

Der Bewertungsmaßstab ist aber nicht durchschaubar. Auf der Suche nach Numerus (36 Treffer) fanden wir beim ersten Mal - in dieser Reihenfolge! - Possessiv-Pronomen und dann Numerus, beide mit 24\%; bei der zweiten Suche am nächsten Tag war die Reihenfolge umgekehrt (vielleicht ein Update?). Die Relevanz von Possessiv-Pronomen scheint daher zu stammen, dass Numerus viermal erwähnt (nicht etwa erklärt) wird (auf der NumerusSeite fünfmal). Bei Modus ist epistemischer Modus (72\%) der erste Treffer; Verbmodus und Satzmodus landen etwa in der Mitte von 11 Einträgen à 7\% deutlich hinter epistemische Minimaleinheit (21\%) und propositionaler Gehalt (14\%).

Bei der Suche ist auch Vorsicht geboten: Diminutiv liefert nichts; man muss lateinisch Diminutivum eingeben. Das ist anfängerfeindlich, zumal naive NutzerInnen sich bei ungewohnten Wortformen sicher gelegentlich vertippen.

Die Liebe zum Lateinischen kann jedoch nicht weit gehen, denn einen Eintrag zu Imperfekt sucht man vergeblich. Das ist bedauerlich, da ja immer noch viele der wenigen, die überhaupt grammatikalische Terminologie kennen, diese am Lateinischen gelernt haben dürften.

Doch die Einträge zu den Tempora sind überhaupt knapp: Futurperfekt, Präsensperfekt (weiterer "thematisch verwandter Begriff": Vergangenheit), Perfekt* $(\rightarrow$ Präsensperfekt), Plusquamperfekt* ( $\rightarrow$ Präteritumperfekt), Präteritumperfekt (verwandt mit: Vorvergangenheit). Futur, Präsens und Präteritum haben keinen Eintrag. Unter Tempus findet man neben einer generellen Charakterisierung von Tempus ,[e]inige Sätze, die sich allein hinsichtlich 
des Tempus unterscheiden" und immerhin einen Link zur Systematischen Grammatik, Absatz Das Tempussystem des Deutschen. Die Ontologie liefert als Hyponyme die einelementigen Termsets Präsens, Futur, Vergangenheitstempus.

$4 \quad$ Ausflüge ins Grüne: Links

Links sind in einem Hypertext-System ausgesprochen wichtig; Grammis ' Ansätze - insbesondere in Bezug auf die Einbindung der Fachbegriffe - sind wiederum sehr gut, doch recht unsystematisch umgesetzt.

\subsection{Ortsverkehr}

Innerhalb der Fachbegriffe selbst scheint ungeregelt, wann und wie oft verlinkt wird; betrachten wir einen Auszug aus dem Eintrag zu Abtönungspartikel:

(4) Abtönungspartikeln sind „satzmodussensitiv“, d.h. es bestehen Bindungen zwischen ein-

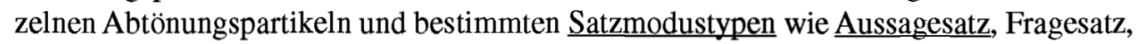
Aufforderungssatz.

Nur Satzmodustypen, nicht aber satzmodussensitiv verweist auf Satzmodus. Ferner ist nur Aussagesatz ( $\rightarrow$ Aussage-Modus, s.u.) verlinkt, obwohl Einträge für Frage-Modus und Aufforderungsmodus (ohne Bindestrich) vorhanden sind. In der Eintragsliste verweist Aussagesatz* auf Aussagesatztyp (,thematisch verwandt“: Aussagesatz und Deklarativsatz). Dort steht:

(5) Ein Satz ist vom Aussagesatztyp, wenn er diese Merkmale aufweist: [...] Typische Sätze vom Typ Aussagesatz sind: [...]

Es geht also nicht um Typen von Aussagesätzen, sondern Aussage(satz) stellt einen (Satz-) Typ dar. Die folgenden Merkmale definieren Aussagesatztyp (im Eintrag Aussagesatztyp zwischen den beiden oben zitierten Sätzen); sie treten fast wortgleich im Eintrag AussageModus auf, den man mit Aussagesatztyp hätte zusammenfassen können. In der zweiten Fassung sind jedoch alle Fachbegriffe außer Imperativ verlinkt, nicht nur zwei.

(6) - Vorfeld vorhanden, jedoch nicht durch eine W-Phrase (z.B. wer, welche ...) belegt

- Verbzweitstellung

- Verbmodus ist nicht Imperativ

- Grenztonmuster fallend

Von den unverlinkten Fachbegriffen kann man immerhin Vorfeld und Imperativ direkt finden, W-Phrase etwas schwieriger. 
Die Lemmaliste ist nämlich nur nach Buchstaben sortiert, ohne Berücksichtigung des Bindestriches. Daher stehen die W-Artikel ganz am Anfang von W. W-Phrase bis W-Relativpronomen stehen hinter Wortverschmelzung $(o<p<r)$. W-Wort* erscheint zweimal ganz am Ende von W und führt einmal zu W-Artikel, einmal zu W-Phrase.

Angesichts dieser verwirrenden Darstellung ist es schade, dass bei W-Phrase und W-Artikel ausnahmsweise kein Link auf die Ontologie gegeben ist (und umgekehrt!). Diese böte nämlich Hilfe: Das Termset $W$-Artikel (zu dem auch noch $w$-Determinativ gehört) ist meronym zum einelementigen Termset W-Phrase.

Doch nicht alles mit W- ist schlecht! In der Erklärung von W-Pronomen findet sich folgender Satz:

(7) andere Bezeichnungen und Zuordnungen: Da die Form verschiedene Funktionen erfüllen kann, wird sie in anderen Darstellungen auch unter „Fragepronomen“ und/oder „Relativpronomen“ angeführt.

Bestand: wer, was, welch-

Derartig klare Anmerkungen sollten bei einer Überarbeitung den Weg weisen.

\subsection{Fernverkehr I: Systematische Grammatik}

In der Systematischen Grammatik scheint zufällig zu sein, ob Verweise innerhalb der Grammatik erfolgen oder in die Fachbegriffsdatenbank zeigen. ${ }^{8}$ Im Abschnitt Der Formenbestand von Indikativ und Konjunktiv etwa wird ausschließlich auf die Begriffsdatenbank verwiesen (vgl. oben (2)). Im Absatz Verbalkomplex hingegen gibt es Verweise innerhalb der Grammatik auf Stellungsfelder und Satzklammer, Hilfsverben, Modalverben u.a.; außerdem stehen in einer Klammer verlinkt: „Tempus, Modus, Genus Verbi, Person und Numerus". Tempus und Modus einerseits führen zu entsprechenden Abschnitten der systematischen Grammatik. Von Genus Verbi, Person und Numerus gelangt man zur Begriffsdatenbank: der Eintrag erscheint dezent in einem Popup-Fenster. ${ }^{9}$ Nur beim Genus Verbi führt wieder ein Verweis in die Grammatik zurück, obwohl auch zu den beiden anderen Begriffen Einträge bestehen (also: Person und Numerus), auf derselben Gliederungs-Ebene wie Das Genus Verbi: Aktiv und Passiv. Die Verlinkung zwischen Grammatik und Fachbegriffen müsste also insgesamt einheitlicher und dichter sein.

8 Ein Link unter Affigierung zu Affix; für naive LeserInnen mag das nicht selbsterklärend sein.

9 Es wird nicht angezeigt, dass der Eintrag aus den Grammatischen Fachbegriffen stammt. Der Navigations-Eintrag ,zurück“ führt im Popup-Fenster zur letzten darin angezeigten Definition (nicht zurück zur Systematischen Grammatik), da er auf die Browser-History des Popup-Fensters zugreift. Ein Zugriff auf die Lemmaliste der Fachbegriffe - etwa um unverlinkte Begriffe nachzuschlagen - ist im Popup auch nicht möglich. 


\subsection{Fernverkehr II: Grammatik in Fragen und Antworten}

Das Modul Grammatik in Fragen und Antworten (GiFA) ist in der Grammis-Navigation das erste und verlinkt in alle Teile von Grammis. Die Fachbegriffe werden wie in der Systematischen Grammatik als Popup angebunden. Der umgekehrte Weg - also von den Fachbegriffen zu GiFA - ist wohl noch nicht vorgesehen.

Die GiFA bietet zwei Suchmasken (neben der Volltextsuche): Fachbegriff suchen und Wortgruppe suchen. Über die erste Maske sucht nicht etwa in den Fachbegriffen, sondern in der GiFA. Offensichtlich wollte man die Suche nach Wortgruppen von der Suche nach Einzelwörtern trennen und hat die Masken unglücklich benannt.

\section{$5 \quad$ Alles eine Frage des Stils, Beispiel: Beispiele}

Die Definitionen sind recht unterschiedlich layoutet; so sind z.B. bei W-Pronomen die erläuternden objektsprachlichen Beispiele kursiv und in Klammern beigefügt. Bei Aussagesatztyp stehen die Beispielsätze mit Korpus-Nachweis als kursive Einzelabsätze. Bei Aussage-Modus kommt hinzu, dass die ersten Beispiele von „(1)“ bis „(3)“ durchnummeriert sind; die abschließenden Beispiele, die in die Erläuterung eingebunden sind, stehen als kursive unnummerierte Einzelsätze. Es mag haarspalterisch erscheinen, aber eine einheitlichere Gestaltung würde erlauben, die Konzentration wohldosierter einzusetzen.

Grammis' Grammatische Fachbegriffe könn(t)en eine große Chance und potentielle Hilfe für linguistische Laien sein. Das System muss jedoch viel laien-freundlicher, vollständiger, durchschaubarer, robuster und hypertextueller werden, damit es seinen Zweck erfüllen kann. Insbesondere die Abgeschlossenheit der Begriffshierarchie und des Definitionsvokabulars sowie die Anbindung an die anderen Teile von Grammis sollten verbessert werden. Am einfachsten sollte sich eine stärkere und systematischere Verlinkung gestalten, die sich wohl evtl. halbautomatisch durchführen ließe.

\section{Literatur}

Fellbaum 1998 = Fellbaum, Christiane (ed.): WordNet: An Electronic Lexical Database . Cambridge, MA: MIT Press, 1998.

Grammis. das grammatische informationssystem des ids. Mannheim: IDS. http://hypermedia.idsmannheim.de/ 